\title{
Craniometrical characteristics of some Sorex araneus chromosomal races
}

\author{
Natalia M. Okulova*, Alexander E. Balakirev \& Victor N. Orlov
}

\begin{abstract}
Skulls of 504 immature common shrews (Sorex araneus) representing seven chromosomal races (Moscow, Lepel, Manturovo, Yagry, Serov, Tomsk, and Neroosa) were examined using 24 morphometric variables and 15 indices derived from them. The races were found to differ in a number of dimensions and proportions of the skull. Each race has its own morphological appearance. Cluster analysis of craniometrical data showed that the Tomsk race was separated from other races. Factor analysis revealed the main trend of craniometrical variability in $S$. araneus chromosomal races. The craniometrical diversity of the species is determined by the variation of characters related to the length of dental rows and the width of the mouth cavity.
\end{abstract}

KEY WORDS: Sorex araneus, chromosome races, skull dimension, craniometric features.

Natalia M.Okulova [natmichok@mtu-net.ru], Alexander E. Balakirev, and Victor N. Orlov, A.N. Severtsov Institute of Ecology and Evolution, Russian Academy of Sciences, Leninskii pr. 33, Moscow 119071, Russia.

\section{Краниометрические характеристики некоторых хромосомных рас Sorex araneus}

\author{
Н.М. Окулова, А.Е. Балакирев, В.Н. Орлов
}

\begin{abstract}
РЕЗЮМЕ. По 24 промерам и 15 индексам проведено сравнение 504 черепов сеголеток обыкновенной бурозубки, относящихся к 7 хромосомным расам. Показано, что расы достоверно отличаются друг от друга по большому числу размерных признаков и пропорций черепа, каждая раса имеет свой морфологический облик. С помощью факторного анализа определено основное направление внутривидовой изменчивости черепа - изменение общих размеров, а также зубных рядов или их отделов (главным образом, длины зубных рядов и ширины ротовой полости).
\end{abstract}

КЛЮЧЕВЫЕ СЛОВА: Sorex araneus, хромосомные расы, размеры черепа, краниометрические особенности.

\section{Introduction}

In European Russia, the common shrew Sorex araneus L., 1758 has almost 20 known chromosomal races formed in this species by Robertsonian fusions from an ancestral acrocentric karyotype (Bulatova et al., 2000; Kozlovsky et al., 2000; Orlov et al., 2004) and it is of interest to compare this variability with other aspects of phenotype and genotype. Craniometric measurements are obvious features for such a comparison. Many authors have studied geographical and population variability in the skull dimensions of $S$. araneus (Dolgov, 1968, 1972, 1985; Senyk, 1973; Sharova, 1975a, b; Puchkovsky, 1981; Yudin, 1989; Gabitova \& Moskvitina, 1992; Bolshakov et al., 1996), but these studies have revealed little geographical patterning except some examples of clinal variation (Dolgov, 1985). Modern methods of multidimensional statistical analysis make a reappraised of craniometrical variation in $S$. araneus worthwhile, particularly within the context of the chromosomal variation within the species.

* Corresponding author
The first investigations comparing craniometry with chromosomal variation in common shrews gave negative results. A canonical discriminant analysis of 8 mandibular dimensions in three chromosomal races from England showed geographic but non-racial distinctions (Searle \& Thorpe, 1987). However, all the interracial hybrids were united in a group separated from the parental races. Sulkava et al. (1985) found that upper dental row lengths (row length, intermediate tooth row length and molar row length) of $S$. araneus from Scandinavia did not relate to chromosome races but reflected geography.

However, more recent investigations have shown that morphological distinctions exist among chromosomal forms in a number of cases. So, Hausser et al. (1991) showed that the Swiss Valais and Vaud races can be separated morphologically. The Valais race was later renamed as $S$. antinorii (Brünner et al., 2002) on the basis of morphological, karyological and biochemical distinctiveness. In the Ural Mountains, 12 populations were subjected to discriminant analysis using four metric and eight craniometric variables and separated into two or three clusters corresponding to the Serov 
race and a group of similar races Sok and Yuruzan (Gabitova \& Moskvitina, 1992; Vasil'ev \& Sharova, 1992). Thus, southern and northern Ural forms are distinguishable. In the same way, the southern Ural populations are separated from Altai populations. By canonical discriminant analysis a clear morphological distinction in the cranial and postcranial skeleton was demonstrated between populations of the Novosibirsk and Tomsk chromosomal races and their hybrids (Polyakov et al., 2002). In one of the most recent studies carried out by analogous methods involving mandibular measurements, little difference was found between Polish chromosomal races that belong to the West and East European karyotypic groups (Wójcik et al., 2000), consistent with data showing an absence of cytochrome $b$ variation between karyotypic groups (WEKG and EEKG) in Poland and also consistent with recent isolation or a bottleneck in their evolution (Ratkiewiczet al., 2002). Polly (2001, 2003, 2007) has investigated differences between populations of $S$. araneus using dental shape variables. He has found small but significant differences between chromosomal races that could not be attributed to geography. Thus, there are varied results from studies of morphometric characteristics in different chromosomal races of common shrew. In particular much needs to be done on races inhabiting European Russia. Therefore, this study examines craniometrical characteristics for seven chromosomal races of Sorex araneus from central and northern regions of European Russia.

The findings in this paper have already been published in Russian (Okulova et al., 2004).

\section{Material and methods}

In this study use was made of 504 skulls of immature common shrews from the Zoological Museum of Moscow University (ZMMU), the Zoology and Ecology Department of Moscow Pedagogic University (MPGU) and the private collection of one of the authors (PC). Animals were captured during summer months from the following localities:

1 - Yagry Island, Severodvinsk, Arkhangelsk Province, 1997-2000, Yagry race*, coll. Balakirev A.E., PC, 54 specimens.

2 - Ramenye Village, Velsk District, Arkhangelsk Province, 1981-1982, Manturovo race*, coll. Kupriyanova I.F. and Nedosekina I.B., MPGU, 53 specimens.

3 - Dan Village, Kortkeros District, Komi Republic, 1981-1983, 1987, Manturovo race, coll. Kupriyanova I.F. and Nedosekina I.B., MPGU, 92 specimens.

4 - Middle Pechora, Komi Republic, 1925, Serov race, coll. Chirkova A., ZMMU, 14 specimens.

5 - Pechoro-Ilichsky Nature Reserve, Komi Republic, 1938, Serov race, coll. is unknown, ZMMU, 22 specimens.

6 - Paleh Village, Ivanovo Province, 2000, Moscow race*, coll. Balakirev A.E., PC, 5 specimens.

7 - Demidovo Village, Pestyaky District, Ivanovo
Province, 1998-2000, Moscow race*, coll. Balakirev A.E. and Shantzeva Yu.V., PC, 25 specimens.

8 - Town Vitchuga, Ivanovo Province, 2000, Moscow race, coll. Balakirev A.E., PC, 2 specimens.

9 - Berezinsky Nature Reserve, Lepel District, Vitebsk Province, Belarus. 1995-2000, Lepel race*, coll. Kashtaljan A.P., ZMMU, 94 specimens.

10 - Gidrouzel Village, Tom-Chumish, Prokopyevsk District, Kemerovo Province. 1957, 1962, Tomsk race, coll. Shtilmark F.R., Okulova N.M., and Koshkina T.V., ZMMU, 52 specimens.

11 - Zvenigorod Biological Station of Moscow State University, Odintzovo District, Moscow Province. 1979, 1997-2001, Moscow race, coll. Shenbrot G.J. et al., ZMMU, 44 specimens.

12 - Dmitrovsk District, Kursk Province, 19261927, 1930, Neroosa race, coll. is unknown, ZMMU, 24 specimens.

13 - Talowaya District, Voronezh Province. 1948, 1989, Neroosa race, coll. Kulukina N.M. and Kovalskaya Yu.M., ZMMU, 12 specimens.

14 - Borovsk District, Voronezh Province, 1919, 1921, 1949, Neroosa race, coll. Obolensky C. and Obraztzov B., ZMMU, 11 specimens.

The localities were chosen to represent chromosomal races established either by direct karyotyping of some specimens (marked by an asterisk) or on the basis of their location within a well-established range. A.I. Kozlovsky carried out the karyotyping of animals from localities 1, 2, 3, 6 and 7. Karyological data from localities 9 and 10 were taken from the literature (Bulatova et al., 2000; Polyakov et al., 2000). N.S. Bulatova and N.A. Shchipanov have identified common shrews from the Pechoro-Ilichsky Nature Reserve as the Serov race (Shchipanov et al., 2005).

A total of 24 measurements were taken (Fig. 1). The measurements were carried out using an ocular micrometer in a binocular microscope MBS to an accuracy of $0.1 \mathrm{~mm}$ for GSL, CBL, BS and HS and to within $0.05 \mathrm{~mm}$ for all other measurements. All measurements are given in $\mathrm{mm}$.

Fifteen indices were calculated from these measurements: 1 - index of skull breadth (ISB $=\mathrm{BS} / \mathrm{CBL}) ; 2$ index of skull height (ISH=HS/CBL); 3 - zygomatic index $(\mathrm{ZY}=\mathrm{ZB} / \mathrm{BS}) ; 4$ - index of the eye-sockets $(\mathrm{IES}=\mathrm{IS} / \mathrm{ZB}) ; 5$ - index of the rostrum $(\mathrm{IR}=\mathrm{BR} / \mathrm{BS})$; 6 - index of the upper premolar dental row (IPR=LIR/ LUR); 7 - facial index (FI=FP/CBL); 8 - index of mandibular height $(\mathrm{IMH}=\mathrm{HM} / \mathrm{LM}) ; 9$ - incisor index $(\mathrm{II}=\mathrm{LLI} / \mathrm{LDR} 1) ; 10$ - molar index $(\mathrm{MY}=\mathrm{LMR} / \mathrm{LDR} 2)$; 11 - index of the length of the mandibular process $(\mathrm{ILMP}=\mathrm{AC} / \mathrm{AA}) ; 12$ - index of the position of the mandibular aperture (IPAP $=\mathrm{IA} / \mathrm{HE}) ; 13$ - index of rostrum length $(\mathrm{IRL}=\mathrm{LR} / \mathrm{ZB}) ; 14$ - relative length of the upper dental row (ILUR=LUR/CBL); 15 - relative length of the lower dental row (ILLR=LDR1/LM).

Immature animals were exclusively used to reduce the influence of age variation. Sexes were not distinguished because it has been shown that sexual dimorphism accounts for the only $2.8 \%$ of morphological 


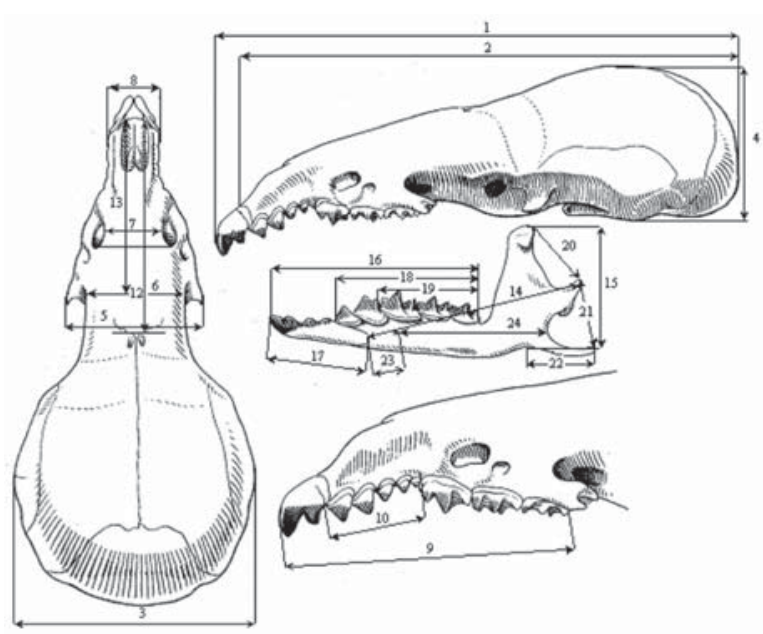

Figure 1. Skull measurements taken (after Yudin, 1989). 1 - skull length (GSL): maximum length with incisors; 2 condylobasal skull length (CBL): the distance from the posterior edge of the occipital bone to the anterior edge of the maxilla; 3 maximum breadth of skull (BS); 4 - maximum height of skull without the tympanic bones (HS); 5 - zygomatic breadth (ZB): the distance between the external edges of the zygomatic arches of the maxilla; 6 - interorbital space (IS): the minimal breadth of the frontal bones in the orbits near their limit with the maxilla; 7 minimal distance between the naso-orbital foramina (DNO); $8-$ breadth of rostrum (BR): the distance between the external edges of the nasal bones level with the first and second intermediate teeth; 9 - length of the upper dental row (LUR): the maximal length of the upper dental row measured from below; 10 - the length of the upper intermediate dental row (LIR) measured from below; 11 the breadth of the osseous palate (BOP); 12 - the length of the facial part of skull (FP): the distance from the anterior edge of the supraorbital foramina to the anterior edge of the maxilla; 13 rostrum length (LR): the distance from the line joining the anterior edges of the naso-orbital foramina to the anterior edge of the maxilla; 14 - maximum length of mandible (LM): the distance from the base of the lower incisor to the top of articular process measured from the lateral side; 15 - the height of mandible (HM) the distance from the top of coronoid process to the edge of the mandible in the region of the angular process measured from the lateral side; 16 - the length of the lower dental row (LDR1): the maximal length of the lower dental row with the incisor, measured from the lateral side, 17 - length of lower incisor (LLI) from the lateral side; 18 - length of the lower dental row Pm+M (LDR2) without the incisors, measured from the lateral side; 19 - length of the lower molar row (LMR) measured from the lateral side, 20 distance between the tips of the condylar and coronoid processes of the mandible (AC); 21 - distance between the tips of the condylar and angular processes of the mandible measured (AA); 22 - length of the angular process (LA); 23 - distance from the base of the incisor to the posterior edge of the mandibular aperture (IA); 24 distance from the hind edge of the mandibular aperture to the posterior edge of the mandible measured between the angular and articular processes (HE)

variability in $S$. araneus while geographical variability is as much as 30 times more (Vasil'ev \& Sharova, 1992). The following abbreviations were used for standard statistical parameters: $M$ - arithmetical mean; $p$ significance level. All standard statistical calculations were carried out using Statistica 6.0.
Seven population samples belonging to five races (330 shrews) were selected to study year-by-year variation in morphology. We used 10 skull measurements to examine this over 2-4 successive years using one-way ANOVAs (Lakin, 1973; Sheffe, 1990; Computer biometrics, 1990).

We also examined the relative influence of chromosomal race and geography on morphology, using twoway ANOVAs. Four populations were selected for study: two of the Neroosa race (Voronezh Province - 39-42 E and Kursk Province $-36-37^{\circ}$ E) and two of the Moscow race (Demidovo Village, Ivanovo Province $39-42^{\circ} \mathrm{E}$ and Zvenigorod Biological Station, Moscow Province $-36-37^{\circ} \mathrm{E}$ ); these populations are remote from one another in a longitudinal direction. Twentytwo skulls from each population were included in the analysis. The influence of factors is symbolized by $\eta$, with a coefficient of determination, $R=\eta^{2}$. The significance of the factors was determined with an $F$-test. We also used cluster analysis with single linkage. The cluster analysis was performed on the means of all 24 variables. Principal components analysis with varimax rotation was also carried out in Statistica 6.0.

\section{Results and discussion}

All measurements and indices displayed significant distinction between races excepting IPAP. Siivonen (1979) regards this index as a species-specific characteristic and our data support this. The characteristics of each race are described below, and the data are given in Tabs 1, 2 and 3.

\section{Western-European karyotypic group}

Moscow race. The common shrews of this race have small longitudinal and vertical skull dimensions, and so ISB and ISH values are large. Distinguishing features are large ZB, BR and BOP, very small GSL, LUR and especially LIR, moderate FP and rather short LR. Length and height of the mandible (LM, HM) are moderate; LDR1 and especially LLI are rather short while LMR is no lower than the average. The angular process is short and the articular process is situated relatively low on the mandible, as a result, AA is very small.

\section{Eastern-European karyotypic group}

Neroosa race. The skull of this race is similar to the Moscow race in general form but is more gracile, characterised by small size overall. The skull breadth is reduced and the ISB is quite large. Just as in the Moscow race, the Neroosa skulls have a large $\mathrm{ZB}$ and $\mathrm{BR}$. BOP is very small, and DNO, IS, FP and LR are even smaller then in the Moscow race. The lengths of dental rows and especially LIR are the shortest of all the races but the relative length of the latter is not extreme. The incisors are short, but relative to the dental row length they are large. The angular process is moderately long and is not noticeably displaced. 


\begin{tabular}{|c|c|c|c|c|c|c|c|c|c|c|c|c|c|c|c|c|c|c|c|c|c|c|c|c|c|c|c|c|}
\hline & $=$ & f & ₹ & f & 7 & & f & f & f & f & f & 7 & $f$ & 8 & f & f & f & f & 7 & $=$ & & $f$ & F & f & 于 & f & 子 & f \\
\hline 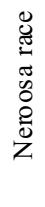 & $\begin{array}{l}\dot{\omega} \\
\dot{\omega} \\
\dot{\Sigma}\end{array}$ & 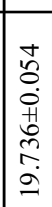 & 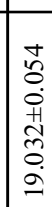 & 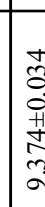 & 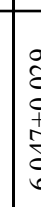 & & 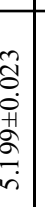 & $\begin{array}{c}\vec{\partial} \\
\overrightarrow{0} \\
\dot{0} \\
\vec{H} \\
\vec{n} \\
\dot{m}\end{array}$ & $\begin{array}{l}0 \\
0 \\
0 \\
0 \\
+1 \\
0 \\
0 \\
i \\
i\end{array}$ & $\mid \begin{array}{l}0 \\
0 \\
0 \\
01 \\
0 \\
\alpha \\
- \\
-1\end{array}$ & 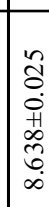 & $\mid \begin{array}{l}\tilde{a} \\
\tilde{a} \\
0 \\
+ \\
+ \\
\alpha \\
\alpha \\
0\end{array}$ & $\begin{array}{l}b \\
b \\
b \\
0 \\
0 \\
\vdots \\
j\end{array}$ & 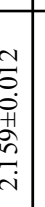 & 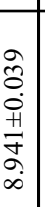 & 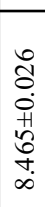 & $\begin{array}{l}0 \\
0 \\
0 \\
0 \\
+1 \\
0 \\
0 \\
\dot{0} \\
\dot{0}\end{array}$ & 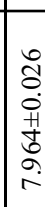 & 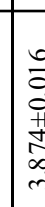 & $\begin{array}{l}2 \\
3 \\
3 \\
3\end{array}$ & & 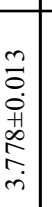 & $\begin{array}{l}\tilde{O} \\
\text { Oे } \\
\dot{1} \\
\ddot{0} \\
\infty \\
\text { i }\end{array}$ & 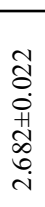 & 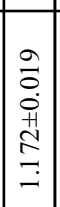 & 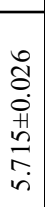 & 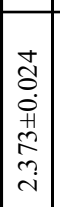 & 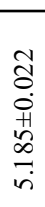 \\
\hline & $=$ & in & 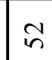 & in & is & v. & $\begin{array}{c}2 \\
n\end{array}$ & n & in & $\approx$ & in & is & & $\sqrt{n}$ & $\bar{n}$ & $\approx$ & in & $\approx$ & in & 8 & in & ñ & in & $\bar{n}$ & $\approx$ & n & $\vec{n}$ & $\bar{n}$ \\
\hline 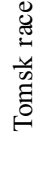 & $\begin{array}{l}\dot{j} \\
\dot{\omega} \\
\dot{\Sigma}\end{array}$ & 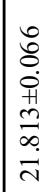 & 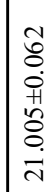 & 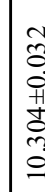 & $\begin{array}{l}\tilde{\varepsilon} \\
z \\
y \\
y\end{array}$ & 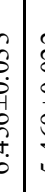 & 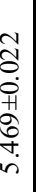 & 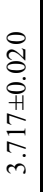 & 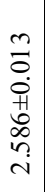 & 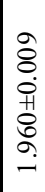 & 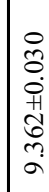 & a & 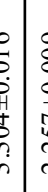 & 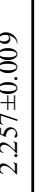 & 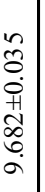 & 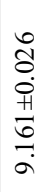 & \begin{tabular}{c}
$\infty$ \\
0 \\
0 \\
0 \\
+1 \\
0 \\
\hdashline-1 \\
$\dot{n}$
\end{tabular} & 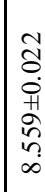 & 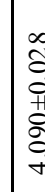 & 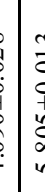 & 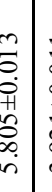 & $\begin{array}{l}\vec{z} \\
\dot{0} \\
\dot{0} \\
\vec{H} \\
\vec{\sigma} \\
\dot{r}\end{array}$ & $\begin{array}{l}\vec{\partial} \\
\dot{0} \\
\dot{1} \\
\text { ते } \\
\vec{m} \\
\dot{m}\end{array}$ & 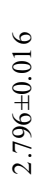 & 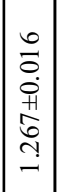 & 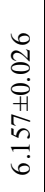 & 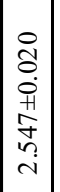 & 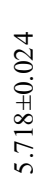 \\
\hline & $=$ & 等 & 哭 & J & 5 & $f$ & $\Xi$ & 学 & 哭 & 哭 & $\stackrel{n}{\exists}$ & $\because g$ & $f$ & 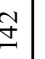 & 品 & $\stackrel{ \pm}{\beth}$ & $\stackrel{+}{ٍ}$ & $\stackrel{\infty}{\sim}$ & 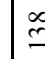 & g & 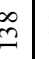 & $\begin{array}{c}\infty \\
\end{array}$ & $\widehat{\Xi}$ & $\stackrel{\infty}{\circ}$ & $\left|\begin{array}{l}\infty \\
\hdashline\end{array}\right|$ & $\hat{m}$ & $\bar{\varrho}$ & 寺 \\
\hline 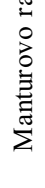 & $\begin{array}{l}\dot{\omega} \\
\dot{\omega} \\
\dot{\Sigma}\end{array}$ & $\begin{array}{l}\tilde{\hat{\theta}} \\
\dot{0} \\
\hat{1} \\
\tilde{0} \\
\hat{\theta} \\
\hat{i}\end{array}$ & $\mid \begin{array}{l}n \\
\tilde{0} \\
0 \\
0 \\
0 \\
0 \\
0 \\
\dot{i} \\
\end{array}$ & 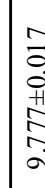 & ఏ & & 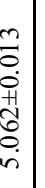 & 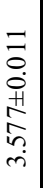 & 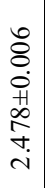 & 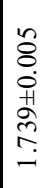 & $\mid \begin{array}{l}n \\
0 \\
0 \\
01 \\
0 \\
0 \\
\infty \\
\infty\end{array}$ & $\mid \begin{array}{l}a \\
\vdots \\
0 \\
\vdots \\
\vdots \\
\vdots\end{array}$ & S. & 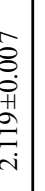 & 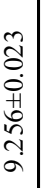 & 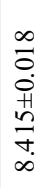 & 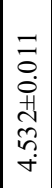 & 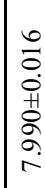 & $\begin{array}{l} \\
\vdots \\
0 \\
0 \\
0 \\
0 \\
c\end{array}$ & $\begin{array}{l}3 \\
3 \\
\vdots\end{array}$ & 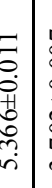 & $\begin{array}{l}\hat{8} \\
\dot{0} \\
+ \\
\tilde{8} \\
\dot{r} \\
\dot{m}\end{array}$ & 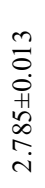 & 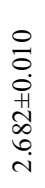 & 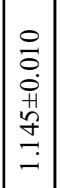 & $\begin{array}{l}0 \\
\dot{0} \\
\dot{0} \\
\dot{+1} \\
\hat{\imath} \\
\dot{0} \\
\text { in }\end{array}$ & 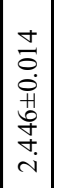 & 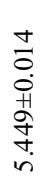 \\
\hline & $=$ & I & I & t & $\delta$ & t & 范 & 吉 & J & Ia & J & J & & 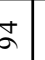 & a & a & J & J & J & 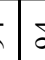 & \pm & a & J & $\stackrel{\infty}{\sim}$ & $\approx$ & $\hat{\sigma}$ & $\stackrel{\infty}{\sim}$ & J \\
\hline 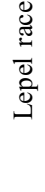 & $\begin{array}{l}\dot{\dot{\omega}} \\
\dot{c} \\
\dot{\Sigma}\end{array}$ & 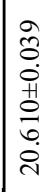 & $\begin{array}{l}\hat{\sigma} \\
\hat{o} \\
0 \\
0 \\
y \\
\sigma \\
\partial \\
\partial\end{array}$ & $\begin{array}{l}\frac{}{2} \\
0 \\
0 \\
+ \\
0 \\
\infty \\
\infty \\
0\end{array}$ & $\begin{array}{l}\vdots \\
\vdots \\
\vdots \\
\vdots\end{array}$ & 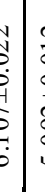 & 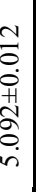 & $\begin{array}{c}\frac{1}{0} \\
0 \\
0 \\
0 \\
0 \\
\vdots \\
m\end{array}$ & 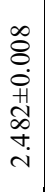 & $\begin{array}{l}\hat{o} \\
0 \\
0 \\
\text { 11 } \\
0 \\
\infty \\
-\end{array}$ & 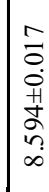 & $\mid \begin{array}{l}\alpha \\
o \\
o \\
\dot{y} \\
\alpha \\
\alpha \\
c\end{array}$ & 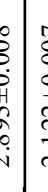 & 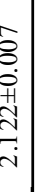 & 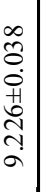 & 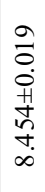 & $\begin{array}{l}m \\
0 \\
0 \\
0 \\
0 \\
0 \\
0 \\
n \\
\sigma \\
\sigma\end{array}$ & $\begin{array}{l}n \\
0 \\
0 \\
+1 \\
+ \\
\infty \\
\infty \\
r\end{array}$ & $\begin{array}{l}\nabla \\
0 \\
0 \\
0 \\
0 \\
0 \\
0 \\
0\end{array}$ & 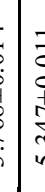 & 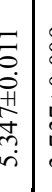 & 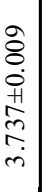 & 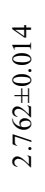 & 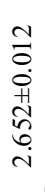 & 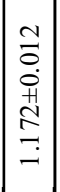 & $\begin{array}{l}\infty \\
0 \\
0 \\
\dot{+1} \\
\hat{\beta} \\
\dot{6} \\
i\end{array}$ & 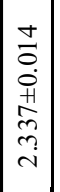 & 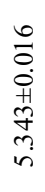 \\
\hline & $=$ & in & i & ते & $\bar{j}$ & & in & r. & r & 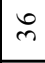 & $\stackrel{m}{m}$ & 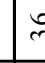 & $: 0$ & $m$ & $m$ & $\bar{m}$ & $\vec{m}$ & $\bar{m}$ & $\bar{m}$ & 5 & $\vec{m}$ & $\vec{m}$ & $\vec{m}$ & $\tilde{\sim}$ & $\stackrel{\text { m }}{2}$ & 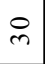 & $\tilde{\lambda}$ & 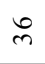 \\
\hline 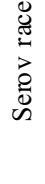 & $\begin{array}{l}\dot{j} \\
\dot{\theta} \\
\dot{\Sigma}\end{array}$ & 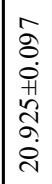 & 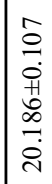 & 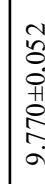 & $\begin{array}{l}7 \\
\\
0 \\
0 \\
0 \\
0\end{array}$ & 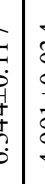 & 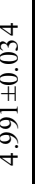 & 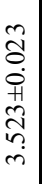 & $\begin{array}{l}\vec{\partial} \\
\overrightarrow{0} \\
\dot{1} \\
\stackrel{0}{0} \\
\vec{n} \\
i\end{array}$ & 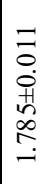 & $\mid \begin{array}{c}0 \\
\delta \\
0 \\
01 \\
0 \\
\infty \\
\infty \\
\infty \\
\infty\end{array}$ & $\begin{array}{l}a \\
0 \\
0 \\
0 \\
0 \\
0\end{array}$ & 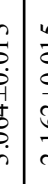 & 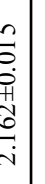 & 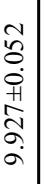 & $\begin{array}{l}\hat{y} \\
0 \\
0 \\
0 \\
0 \\
\hat{n} \\
\infty \\
\infty\end{array}$ & $\begin{array}{c}0 \\
\tilde{o} \\
0 \\
\dot{1} \\
\ddot{n} \\
\sigma \\
\tilde{r} \\
+\end{array}$ & $\mid \begin{array}{l}\frac{y}{0} \\
0 \\
\dot{1} \\
\infty \\
\frac{\alpha}{\infty} \\
\end{array}$ & 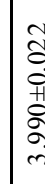 & $\begin{array}{l}z \\
\vdots \\
3 \\
3\end{array}$ & 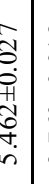 & 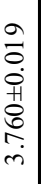 & 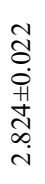 & $\begin{array}{l}\overrightarrow{5} \\
0 \\
0 \\
+ \\
+ \\
\infty \\
n \\
i\end{array}$ & 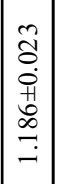 & $\begin{array}{l}\vec{y} \\
\dot{0} \\
\dot{+} \\
+ \\
\text { f. } \\
\dot{b}\end{array}$ & 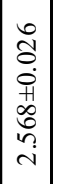 & 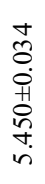 \\
\hline & $=$ & 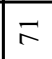 & $\approx$ & 8 & ก & & $\bumpeq 1$ & 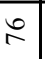 & $\cong$ & $\stackrel{0}{\sim}$ & $\underset{I}{I}$ & 17 & 0 & $p$ & 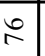 & $\therefore$ & $\stackrel{ \pm}{\sim}$ & $g$ & A & 17 & \pm & $n$ & $\stackrel{\nabla}{\sim}$ & $\approx$ & $\approx$ & $\approx$ & $\approx$ & $\because$ \\
\hline 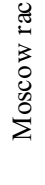 & $\begin{array}{l}\dot{j} \\
\dot{\omega} \\
\dot{\Sigma}\end{array}$ & $\begin{array}{l}\hat{\tilde{o}} \\
\dot{0} \\
\hat{1} \\
\hat{\alpha} \\
\hat{\sigma} \\
2\end{array}$ & 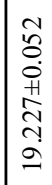 & 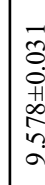 & $\begin{array}{l}\vdots \\
\vdots \\
0 \\
\vdots \\
0 \\
\vdots \\
b\end{array}$ & 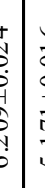 & 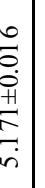 & 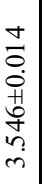 & $\begin{array}{l}\vec{a} \\
0 \\
0 \\
\vec{H} \\
\vec{n} \\
i\end{array}$ & 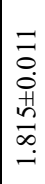 & $\mid \begin{array}{l}\vec{\delta} \\
0 \\
0 \\
\infty \\
\delta \\
\infty \\
\infty\end{array}$ & $\mid \begin{array}{c}a \\
a \\
0 \\
\vdots \\
\vdots \\
\alpha \\
0\end{array}$ & 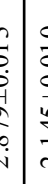 & 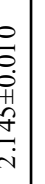 & 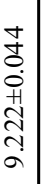 & 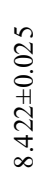 & $\begin{array}{l}n \\
0 \\
0 \\
0 \\
0 \\
0 \\
0 \\
0 \\
+ \\
+ \\
\end{array}$ & 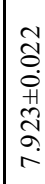 & $\begin{array}{l}n \\
0 \\
0 \\
0 \\
\tilde{c} \\
\infty \\
m\end{array}$ & 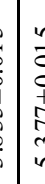 & $\begin{array}{l}n \\
0 \\
0 \\
0 \\
1 \\
\vdots \\
0 \\
n \\
n\end{array}$ & 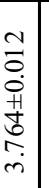 & 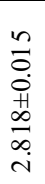 & 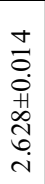 & 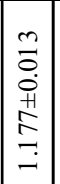 & 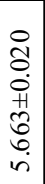 & $\left|\begin{array}{c}\overrightarrow{1} \\
0 \\
0 \\
\dot{1} \\
\hat{2} \\
\hat{n} \\
i\end{array}\right|$ & 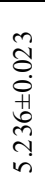 \\
\hline & $=$ & in & $\approx$ & in & $\pi$ & 65 & $\hat{n}$ & $n$ & $\hat{n}$ & $\hat{n}$ & $\approx$ & 18 & 8 & 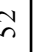 & $n$ & $n$ & 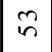 & $\tilde{n}$ & in & 35 & $\hat{n}$ & $\therefore$ & $n$ & in & $\tilde{n}$ & $\hat{n}$ & $\approx$ & $n$ \\
\hline 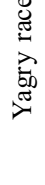 & $\begin{array}{l}\dot{\dot{j}} \\
\dot{\vec{n}}\end{array}$ & 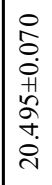 & 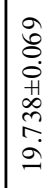 & $\begin{array}{l}0 \\
0 \\
0 \\
0 \\
0 \\
0 \\
0 \\
0\end{array}$ & 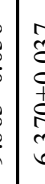 & 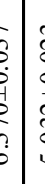 & 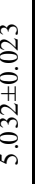 & 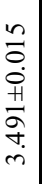 & 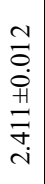 & $\begin{array}{l}\vec{\Xi} \\
0 \\
\dot{0} \\
\stackrel{2}{\rho} \\
\stackrel{-}{-}\end{array}$ & $\begin{array}{l}0 \\
0 \\
0 \\
0 \\
\infty \\
0 \\
0 \\
\infty \\
\infty\end{array}$ & $\begin{array}{l}\infty \\
0 \\
0 \\
\vdots \\
\vdots \\
\vdots \\
0\end{array}$ & 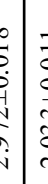 & 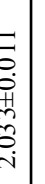 & 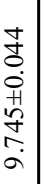 & $\begin{array}{l}\hat{\tilde{o}} \\
\hat{0} \\
\hat{1} \\
\hat{0} \\
\sim \\
\infty \\
\infty\end{array}$ & 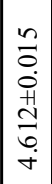 & 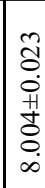 & 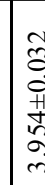 & $\begin{array}{lll}3 \\
3 \\
3 \\
4 \\
4 \\
3\end{array}$ & $\begin{array}{c}m \\
0 \\
0 \\
0 \\
0 \\
0 \\
0 \\
m \\
m \\
n \\
n\end{array}$ & 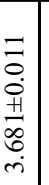 & 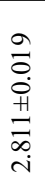 & 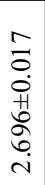 & $\mid \begin{array}{c}\vec{a} \\
0 \\
0 \\
\dot{0} \\
\stackrel{0}{0} \\
\stackrel{-}{-}\end{array}$ & 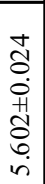 & 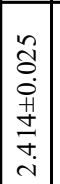 & 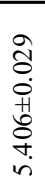 \\
\hline$\overline{\bar{z}}$ & & हु & $\ddot{\theta}$ & $\tilde{m}$ & $\underline{g}$ & & กै & $\Omega$ & z & $\widetilde{m}$ & 3 & 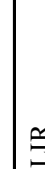 & 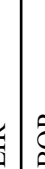 & o & 空 & 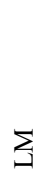 & 至 & ב & $\Xi$ & G & $\overrightarrow{3}$ & 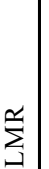 & 䢴 & $\mathbb{4}$ & $\unlhd$ & 馬 & $\unlhd$ & $\mathfrak{G}$ \\
\hline
\end{tabular}


Table 2. The means (M) and standard errors (s.e.) for skull indices in the different chromosome races of

S. araneus.

\begin{tabular}{|c|c|c|c|c|c|c|c|c|}
\hline \multirow{2}{*}{$\begin{array}{l}\text { Skull } \\
\text { measurements }\end{array}$} & \multicolumn{2}{|c|}{ Yagry race } & \multicolumn{2}{|c|}{ Moscow race } & \multicolumn{2}{|c|}{ Serov race } & \multicolumn{2}{|c|}{ Lepel race } \\
\hline & $\mathrm{M} \pm$ s.e. & $\mathrm{n}$ & $\mathrm{M} \pm$ s.e. & $\mathrm{n}$ & $\mathrm{M} \pm$ s.e. & $\mathrm{n}$ & $\mathrm{M} \pm$ s.e. & $\mathrm{n}$ \\
\hline ISB & $0.490 \pm 0.002$ & 54 & $0.499 \pm 0.001$ & 68 & $0.484 \pm 0.002$ & 27 & $0.494 \pm 0.001$ & 94 \\
\hline ISH & $0.319 \pm 0.002$ & 54 & $0.329 \pm 0.001$ & 72 & $0.316 \pm 0.006$ & 28 & $0.307 \pm 0.001$ & 94 \\
\hline $\mathrm{ZY}$ & $0.522 \pm 0.003$ & 54 & $0.542 \pm 0.002$ & 66 & $0.515 \pm 0.004$ & 29 & $0.518 \pm 0.001$ & 94 \\
\hline IES & $0.700 \pm 0.004$ & 54 & $0.686 \pm 0.003$ & 73 & $0.706 \pm 0.005$ & 36 & $0.690 \pm 0.003$ & 94 \\
\hline IR & $0.182 \pm 0.001$ & 54 & $0.191 \pm 0.002$ & 69 & $0.184 \pm 0.002$ & 29 & $0.184 \pm 0.001$ & 94 \\
\hline IPR & $0.342 \pm 0.002$ & 54 & $0.334 \pm 0.001$ & 73 & $0.347 \pm 0.002$ & 35 & $0.337 \pm 0.001$ & 93 \\
\hline FI & $0.494 \pm 0.002$ & 54 & $0.479 \pm 0.002$ & 72 & $0.493 \pm 0.003$ & 30 & $0.463 \pm 0.002$ & 94 \\
\hline IMH & $0.556 \pm 0.003$ & 54 & $0.547 \pm 0.002$ & 74 & $0.534 \pm 0.003$ & 31 & $0.540 \pm 0.002$ & 94 \\
\hline II & $0.491 \pm 0.003$ & 54 & $0.484 \pm 0.002$ & 74 & $0.487 \pm 0.002$ & 31 & $0.479 \pm 0.001$ & 94 \\
\hline MY & $0.688 \pm 0.001$ & 53 & $0.670 \pm 0.002$ & 74 & $0.689 \pm 0.002$ & 31 & $0.699 \pm 0.001$ & 94 \\
\hline ILMP & $1.043 \pm 0.010$ & 53 & $1.075 \pm 0.008$ & 71 & $1.131 \pm 0.065$ & 23 & $1.044 \pm 0.008$ & 78 \\
\hline IPAP & $0.202 \pm 0.003$ & 54 & $0.208 \pm 0.003$ & 72 & $0.206 \pm 0.004$ & 30 & $0,226 \pm 0.019$ & 93 \\
\hline IRL & $10.372 \pm 0.055$ & 54 & $10.300 \pm 0.047$ & 76 & $10.464 \pm 0.065$ & 36 & $10.259 \pm 0.031$ & 94 \\
\hline ILUR & $0.439 \pm 0.002$ & 54 & $0.450 \pm 0.001$ & 70 & $0.439 \pm 0.002$ & 29 & $0.432 \pm 0.001$ & 93 \\
\hline \multirow[t]{3}{*}{ ILLR } & $0.962 \pm 0.003$ & 54 & $0.941 \pm 0.003$ & 74 & $0.953 \pm 0.005$ & 31 & $0.932 \pm 0.002$ & 94 \\
\hline & \multicolumn{2}{|c|}{ Manturovo race } & \multicolumn{2}{|l|}{ Tomsk race } & \multicolumn{2}{|c|}{ Neroosa race } & & \\
\hline & $\mathrm{M} \pm$ s.e. & $\mathrm{n}$ & $\mathrm{M} \pm$ s.e. & $\mathrm{n}$ & $\mathrm{M} \pm$ s.e. & $\mathrm{n}$ & & \\
\hline ISB & $0.487 \pm 0.001$ & 144 & $0.490 \pm 0.002$ & 51 & $0.493 \pm 0.002$ & 47 & & \\
\hline ISH & $0.310 \pm 0.001$ & 145 & $0.307 \pm 0.001$ & 52 & $0.318 \pm 0.002$ & 47 & & \\
\hline ZY & $0.518 \pm 0.001$ & 140 & $0.531 \pm 0.002$ & 51 & $0.555 \pm 0.003$ & 47 & & \\
\hline IES & $0.708 \pm 0.002$ & 141 & $0.680 \pm 0.004$ & 52 & $0.676 \pm 0.004$ & 47 & & \\
\hline IR & $0.178 \pm 0.001$ & 144 & $0.190 \pm 0.001$ & 51 & $0.203 \pm 0.001$ & 47 & & \\
\hline IPR & $0.345 \pm 0.001$ & 145 & $0.353 \pm 0.001$ & 51 & $0.334 \pm 0.001$ & 47 & & \\
\hline FI & $0.462 \pm 0.001$ & 145 & $0.461 \pm 0.001$ & 51 & $0.470 \pm 0.002$ & 47 & & \\
\hline IMH & $0.539 \pm 0.002$ & 129 & $0.564 \pm 0.002$ & 52 & $0.550 \pm 0.002$ & 47 & & \\
\hline II & $0.489 \pm 0.001$ & 138 & $0.478 \pm 0.003$ & 52 & $0.487 \pm 0.002$ & 47 & & \\
\hline MY & $0.690 \pm 0.001$ & 138 & $0.676 \pm 0.001$ & 52 & $0.698 \pm 0.001$ & 46 & & \\
\hline ILMP & $1.037 \pm 0.007$ & 95 & $1.012 \pm 0.009$ & 51 & $1.072 \pm 0.013$ & 42 & & \\
\hline IPAP & $0.213 \pm 0.013$ & 137 & $0.206 \pm 0.003$ & 52 & $0.206 \pm 0.004$ & 47 & & \\
\hline IRL & $10.463 \pm 0.028$ & 144 & $10.965 \pm 0.045$ & 51 & $10.370 \pm 0.043$ & 47 & & \\
\hline ILUR & $0.433 \pm 0.001$ & 145 & $0.446 \pm 0.001$ & 51 & $0.454 \pm 0.001$ & 47 & & \\
\hline ILLR & $0.950 \pm 0.002$ & 133 & $0.935 \pm 0.003$ & 52 & $0.941 \pm 0.003$ & 47 & & \\
\hline
\end{tabular}

Lepel race. Animals of this race have a moderately long skull, which is broad and flat, with a moderately long zygomatic arch, but with wide eye-sockets and rostrum. The absolute and relative lengths of the facial part of the skull are small. The mandibles are of moderate length; the lower dental row and especially the lower incisors are short; otherwise the length of the molar row is moderate. The angular process is rather short and the articular process is slightly raised. As a result both $\mathrm{AC}$ and $\mathrm{AA}$ are very small.
Manturovo race. A rather long skull with moderate breadth and height characterises the Manturovo race. The zygomatic breadth is rather small and the interorbital breath quite large. The rostrum and facial part of skull are of moderate length but the former is rather narrow. The upper dental row is of average size. The length of the lower dental row and the lower incisor are moderate but the lower molar dental row is slightly shortened especially at the expense of the premolars. The angular process is of average size while the articular process is somewhat larger than normal. 
Table 3. Influence of year-by-year variation $\left(\eta^{2}\right)$ on craniometric characters in different races of common shrew from specified sites.

\begin{tabular}{|l|c|c|c|c|c|c|c|}
\hline \multirow{2}{*}{ Measurements } & Yagry race & Manturovo race & \multicolumn{2}{|c|}{ Moscow race } & Lepel race & \multicolumn{2}{c|}{ Neroosa race } \\
\cline { 2 - 8 } & $\begin{array}{c}\text { Yagry Island, } \\
\mathrm{n}=50\end{array}$ & $\begin{array}{c}\text { Dan, } \\
\mathrm{n}=92\end{array}$ & $\begin{array}{c}\text { Demidovo, } \\
\mathrm{n}=19\end{array}$ & $\begin{array}{c}\text { Zvenigorod } \\
\text { Biological Station, } \\
\mathrm{n}=41\end{array}$ & $\begin{array}{c}\text { Berezinsky } \\
\text { Nature Reserve, } \\
\mathrm{n}=87\end{array}$ & $\begin{array}{c}\text { Kursk } \\
\text { District, } \\
\mathrm{n}=24\end{array}$ & $\begin{array}{c}\text { Voronezh } \\
\text { District, } \\
\mathrm{n}=17\end{array}$ \\
\hline GSL & $0.179^{* *}$ & $\mathrm{~ns}$ & $\mathrm{~ns}$ & $0.183^{* * *}$ & $\mathrm{~ns}$ & $\mathrm{~ns}$ & $\mathrm{~ns}$ \\
\hline CBL & $0.188^{* *}$ & $\mathrm{~ns}$ & $\mathrm{~ns}$ & $0.304^{* *}$ & $\mathrm{~ns}$ & $0.551^{* * *}$ & $\mathrm{~ns}$ \\
\hline LUR & $0.126^{*}$ & $\mathrm{~ns}$ & $\mathrm{~ns}$ & $0.267^{* *}$ & $\mathrm{~ns}$ & $\mathrm{~ns}$ & $\mathrm{~ns}$ \\
\hline HS & $\mathrm{ns}$ & $0.09^{*}$ & $\mathrm{~ns}$ & $0.306^{*}$ & $\mathrm{~ns}$ & $\mathrm{~ns}$ & $\mathrm{~ns}$ \\
\hline ZB & $\mathrm{ns}$ & $0.107^{*}$ & $\mathrm{~ns}$ & $0.214^{*}$ & $\mathrm{~ns}$ & $\mathrm{~ns}$ & $\mathrm{~ns}$ \\
\hline BS & $0.227^{* *}$ & $\mathrm{~ns}$ & $\mathrm{~ns}$ & $0.154^{*}$ & $\mathrm{~ns}$ & $0.333^{*}$ & $\mathrm{~ns}$ \\
\hline LM & $\mathrm{ns}$ & $\mathrm{ns}$ & $0.102^{*}$ & $0.201^{*}$ & $\mathrm{~ns}$ & $\mathrm{~ns}$ & $\mathrm{~ns}$ \\
\hline LIR & $\mathrm{ns}$ & $\mathrm{ns}$ & $\mathrm{ns}$ & $\mathrm{ns}$ & $\mathrm{ns}$ & $\mathrm{ns}$ & $\mathrm{ns}$ \\
\hline BR & $\mathrm{ns}$ & $\mathrm{ns}$ & $\mathrm{ns}$ & $\mathrm{ns}$ & $0.214^{* * *}$ & $\mathrm{~ns}$ & $\mathrm{~ns}$ \\
\hline BOP & $\mathrm{ns}$ & $\mathrm{ns}$ & $\mathrm{ns}$ & $\mathrm{ns}$ & $\mathrm{ns}$ & $\mathrm{ns}$ & $\mathrm{ns}$ \\
\hline
\end{tabular}

$\mathrm{n}$ - number of individuals; ns - not significant; * $p<0.05, * * p<0.01, * * * p<0.001$

Yagry race. This is a newly described race with diagnostic chromosomes go, hi, kq, $m p, n r$ (Orlov et al., 2004). It is characterised by the following traits: a moderately long skull with principal longitudinal dimensions (GSL, CBL, LUR, LIR and LDR1) of average length, but transverse measurements (BS, ZB, BR and especially IS, DNO and BOP) comparatively narrow. The height of the skull (ISH) is large. Long lower incisors and an extended facial part of skull with a small rostrum length are race-specific features. The height of the mandible and the length of lower dental row are small. ILMP is rather large, the mandibular processes are moderate. The dimensions of the upper dental row are also moderate.

\section{Ural races}

Serov race. This race has a very long, moderately wide and rather high skull which narrows at the eyesockets. The extremely long facial part of skull is also characteristic. The values for ZB and IS are moderate and the rostrum has a moderate length and width. The upper dental row and BOP are long as is the mandible, although the height is moderate. The lower dental row is absolutely rather long and its relative length is the greatest among the all races studied. The premolars are small while the length of the lower incisors is moderate. The length of the angular process is extremely large in comparison to other races but the articular process is displaced downward as in the Moscow race.

\section{Siberian races}

Tomsk race. Shrews of this race have a larger skull then all other races examined. The facial part of skull is relatively rather short and the rostrum is wide. The length of the upper dental row (especially the premolars) is relatively large. The mandible is relatively high and relative length of the lower molar row is low. The premolars are rather large. The angular process is extended.

In the light of our new data, it is interesting to reconsider the result of V.A. Dolgov $(1968,1985)$. Although he made three measurements only (CBL, LUR and BR) he showed that the large skulled populations from Altai and Sayan were different from the wide-nosed populations from Western Siberia and the Ural mountains, which in turn were distinct from the Karelian populations, which were characterised by rather small skulls, and those from South-Western Europe with moderate large skulls, very long dental rows and a wide rostrum.

The influence of year-by-year variation on skull dimensions of Sorex araneus. As shown in Tab. 3, annual variation was significant for 7 of 10 measurements in the Zvenigorod sample (Moscow race) and for 4 of 10 in the Yagry race. However, very little of the morphological variation is accounted for year-to-year variation, not more than $6 \%$. Based on these findings, we pooled data from all years for racial comparison.

The influence of race and geographical location on variability. ANOVAs revealed that geographical location is not significant for any measurements. On the other hand, race affected 8 out of 10 measurements studied (all except LUR and BS, Tab. 4).

Thus, we conclude that only race significantly influenced variation, not geography as such. This result can be tested by including more distant populations in the analysis. However, we imagine that clinal geographical variability in $S$. araneus results from the differences which characterise the chromosome races. 
Table 4. Impact of chromosome race $\left(\eta^{2}\right)$ on craniometric measurements.

\begin{tabular}{|l|c|c|c|}
\hline \multirow{2}{*}{ Dimensions } & \multicolumn{3}{|c|}{ Parameters of analysis } \\
\cline { 2 - 4 } & $\eta^{2}$ & $\mathrm{~F}$ & $p$ \\
\hline GSL & 0.483 & 24.17 & $<0.0001$ \\
\hline CBL & 0.286 & 11.12 & $<0.0001$ \\
\hline LUR & & & $\mathrm{ns}$ \\
\hline BS & & & $\mathrm{ns}$ \\
\hline BOP & 0.102 & 3.15 & $<0.05$ \\
\hline FP & 0.535 & 28.11 & $<0.0001$ \\
\hline HS & 0.399 & 14.65 & $<0.0001$ \\
\hline BR & 0.421 & 19.28 & $<0.0001$ \\
\hline LM & 0.391 & 18.74 & $<0.0001$ \\
\hline LIR & 0.084 & 2.88 & $<0.05$ \\
\hline
\end{tabular}

ns - not significant

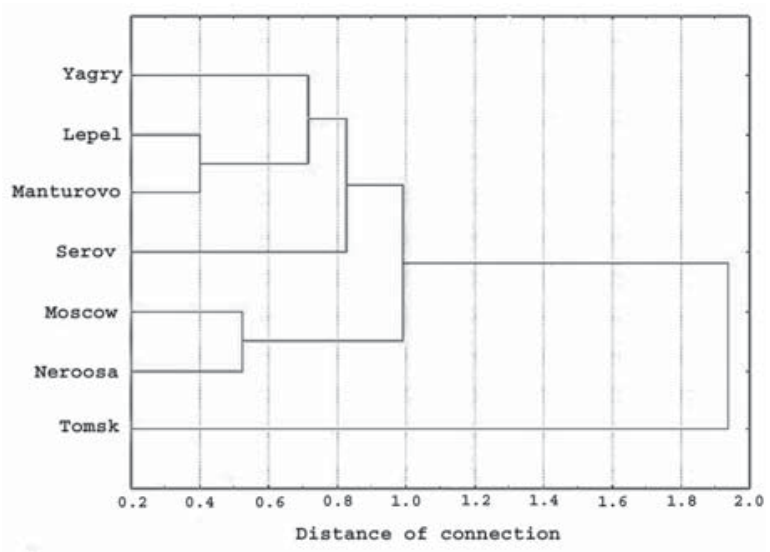

Figure 2. Relationships of different chromosome races of common shrew based on 24 skull variables.

Analysis of interracial relationships. The results of a cluster analysis of 24 mean skull dimensions are presented in Fig. 2. The Tomsk race is distinct from all other chromosomal races, which form two clusters. One cluster consists of the Moscow and Neroosa races. In the other cluster, the Lepel and Manturovo races are situated closest each other, the Yagry race is next closest, and the Serov race furthest of all.

We used principal components analysis on the 24 skull measurements. For simplicity, only the variables which had factor loadings of 0.7 and above are included in Tab. 5. These were GSL, CBL, BR and LUR; overall, factors I and II described $89.09 \%$ of the variation. Factor 1 includes skull length (GSL and CBL) and length of the lower dental row, and Factor 2 includes rostral breadth (BR). These data suggest that chromosome race differentiation is reflected by general skull length and facial breadth.

In principal component space, the two European races (Neroosa and Moscow) are almost completely
Table 5. Factor loading in the principal components analysis.

\begin{tabular}{|l|c|c|}
\hline Dimensions & Factor 1 & Factor 2 \\
\hline GSL & 0.970 & 0.080 \\
\hline CBL & 0.958 & -0.031 \\
\hline BR & 0.018 & 0.969 \\
\hline LUR & 0.716 & 0.495 \\
\hline Cumulative value & 3.073 & 1.048 \\
\hline Dispersion explained (\%) & 62.67 & 26.42 \\
\hline $\begin{array}{l}\text { Dispersion explained } \\
\text { (Overall \%) }\end{array}$ & \multicolumn{2}{|c|}{89.09} \\
\hline
\end{tabular}

separated from the Tomsk race (Fig. 3). The Neroosa and Moscow races also formed a separate cluster in cluster analysis. Races such as Lepel and Manturovo overlap with the Tomsk race only slightly. The principal component approach confirmed the large distance of the Tomsk race from other races as indicated in the cluster analysis. This confirms earlier findings that the Siberian common shrews are distinct from EuropeanUral shrews; indeed the former are considered as a separate subspecies (Yudin, 1989).

The distinctive features identified here using craniometry emphasise the fact that the races are not only chromosomal categories, but are also distinct intraspecific groups marked by a complex of features including skull shape. In conclusion, craniometry can allow researchers to distinguish chromosome races of common shrew. Further studies are needed to accurately determine relationships of chromosomal races based on this method.

ACKNOWLEDGMENTS. We thank Alexander I. Kozlovsky for karyotyping of shrews. The work was supported by the Russian Foundation for Basic Research (grants Nos. 00-04-49142 and 03-04-06673Mac). We also are grateful to Dr. P.D. Polly for his valuable comments on the manuscript.

\section{References}

Bolshakov V.N., Vasil'ev A.G. \& Sharova L.P. 1996. [The fauna and population ecology of shrews from Ural Mts (Mammalia, Soricidae)] // Trudy Uchenyh UrO RAN, Seria Biologia. Ekaterinburg: Institute of Animal and Plant Ecology, Ural Branch of the Russian Academy of Sciences. P.1-267 [in Russian].

Brünner H., Lugon-Moulin N., Balloux F., Fumagalli L. \& Hausser J. 2002. A taxonomical re-evaluation of the Valais chromosome race of the common shrew Sorex araneus (Insectivora: Soricidae) // Acta Theriologica. Vol.47. Suppl.1. P.245-275.

Bulatova N., Searle J.B., Bystrakova N. Nadjafova R., Shchipanov N. \& Orlov V. 2000. The diversity of chromosome races in Sorex araneus from European Russia // Acta Theriologica. Vol.45. Suppl.1. P.33-46.

[Computer biometrics]. 1990. Nosov V.N. (ed.). Moskva: Izdatel'stvo Moskovskogo Gosudarstvennogo Univer- 


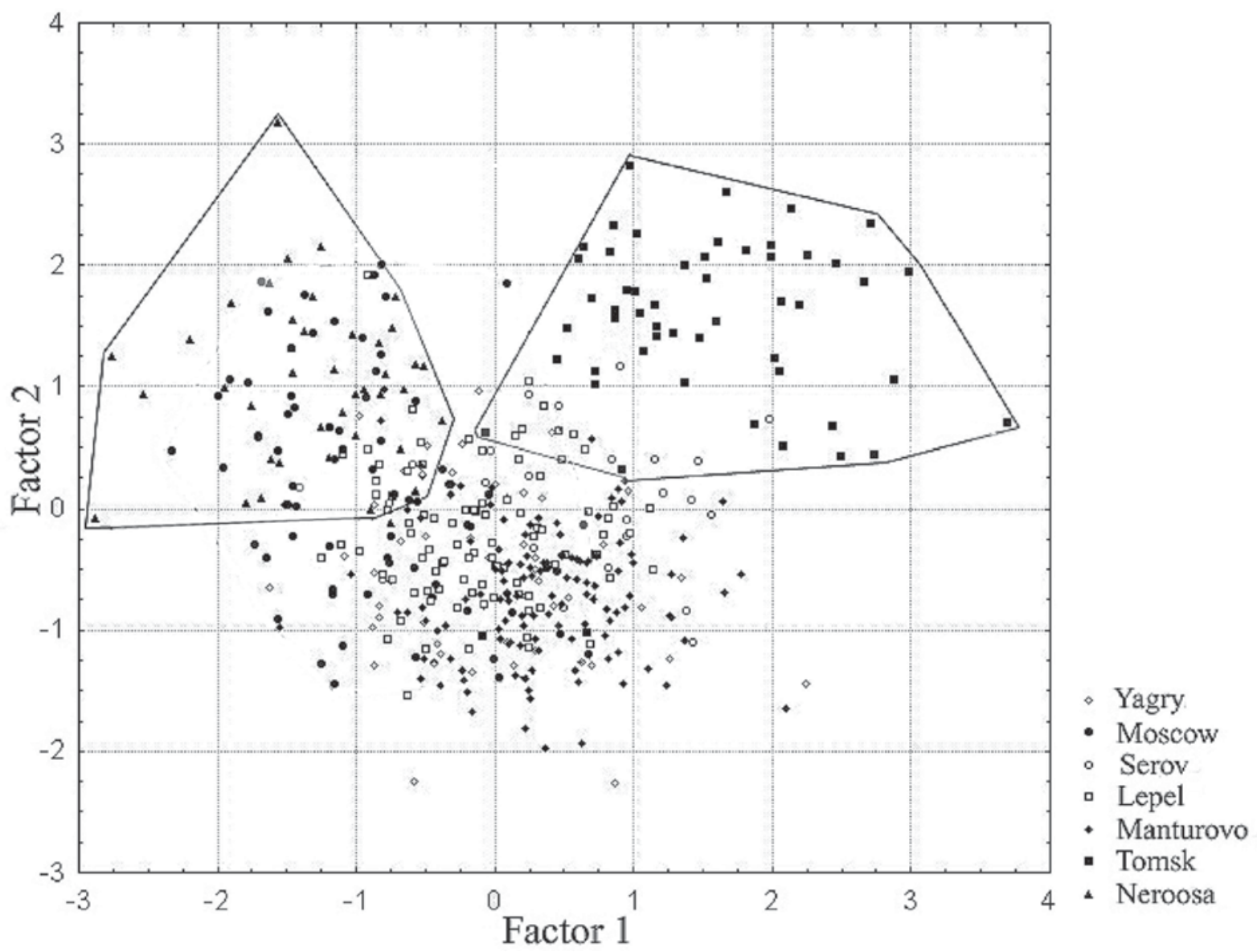

Figure 3. The distribution of specimens of common shrew belonging to specified chromosomal races along two principal component axes calculated from 24 skull variables.

siteta. [In Russian].

Dolgov V.A. 1968. [The peculiarities and variability of odontological features of Palearctic shrews (Mammalia, Sorex)] // Trudy Zoologicheskogo Muzeya MGU. Vol.10. P.179-199 [in Russian].

Dolgov V.A. 1972. [Craniometry and patterns of geographical variability of craniological features of Palearctic shrews (Mammalia, Sorex)] // Trudy Zoologicheskogo Muzeya MGU. Vol.13. P.150-186 [in Russian, with English summary].

Dolgov V.A. 1985. [The shrews of Old World]. Moskva: Izdatel'stvo Moskovskogo Gosudarstvennogo Universiteta. [In Russian].

Gabitova A.T. \& Moskvitina N.S. 1992. [Intra- and interpopulation variability of craniometric characters in common shrew (Sorex araneus L.) in chromosomally monoand polymorphic populations] // Morfologicheskaya i Khromosomnaya Izmenchivost' Melkikh Mlekopitayuschikh. Ekaterinburg: Nauka, Ural'skoe Otdelenie. P.1136 [in Russian].

Hausser J., Bosshard F., Taberlet P. \& Wójcik J. 1991. Relationships between chromosome races and species of Sorex araneus group in the Western Alps // Mémoires de la Société Vaudoise des Sciences Naturelles. Vol.19. P.79-95.
Kozlovsky A., Orlov V., Okulova N., Kovalskaya J. \& Searle J. B. 2000. Chromosome studies of common shrew from northern and central parts of European Russia // Acta Theriologica. Vol.45. Suppl.1. P.27-31.

Lakin G.F. 1973. [Biometrics]. Moskva: Vysschaya Shkola. [In Russian].

Okulova N.M., Balakirev A.E. \& Orlov V.N. 2004. [Craniometrical characteristics of some Sorex araneus (Insectivora) chromosomal races] // Zoologicheskii Zhurnal. Vol.83. P.1476-1487 [in Russian, with English summary].

Orlov V.N., Bulatova N.Sh., Kozlovsky A.I. \& Balakirev A.E. 2004. [Hierarchy of intraspecific taxa of the common shrew Sorex araneus L. (Insectivora), and taxonomic structure of species in mammals] // Zoologicheskii Zhurnal. Vol.83. P.199-212 [in Russian, with English summary].

Polly P.D. 2001. On morphological clocks and paleophylogeography: Towards a timescale for Sorex hybrid zones // Genetica. Vol.112-113. P.339-357.

Polly P.D. 2003. Paleophylogeography of Sorex araneus: molar shape as a morphological marker for fossil shrews // Mammalia. Vol.67. P.233-243.

Polly P.D. 2007. Phylogeographic differentiation in Sorex araneus: morphology in relation to geography and kary- 
otype // Russian Journal of Theriology. Vol.6. No.1. P.73-84.

Polyakov A.V., Zima J., Searle J.B., Borodin P.M. \& Ladygina T. 2000. Chromosome races of the common shrew Sorex araneus in the Ural Mts: a link between Siberia and Scandinavia? // Acta Theriologica. Vol.45. Suppl.1. P.19-26.

Polyakov A.V., Panov V.V., Ladygina T.Yu., Bochkarev M.N., Rodionova M.I. \& Borodin P.M. 2001. [The chromosome evolution of common shrew Sorex araneus L. within postglacial period on South Ural and Siberia] // Genetika. Vol.37. P.448-455 [in Russian, with English summary].

Polyakov A.V., Onischenko S.S., Ilyashenko V.B, Searle J.B. \& Borodin P.M. 2002. Morphometric difference between the Novosibirsk and Tomsk chromosome races of Sorex araneus in a zone of parapatry // Acta Theriologica. Vol.47. Suppl.1. P.381-387.

Puchkovsky S.V. 1981. [The morphophysical description of present-year adult male of common shrew] // Fauna i Ekologiya Zhivotnykh UASSR i Prilegayushchikh Raionov. Izhevsk: Izdatel'stvo Udmurtskogo Universiteta. P.88-94 [in Russian].

Ratkiewicz M., Fedyk S., Banaszek A., Chętnicki W., Szałaj K., Gielly L. \& Taberlet P. 2002. The evolutionary history of the two karyotypic groups of the common shrew, Sorex araneus in Poland // Heredity. Vol.88. P.235-242.

Senyk A.F. 1972. [Common shrew (Sorex araneus L.) of Ukrainian Carpathian Mts] // Vestnik Zoologii. Vol.3. P.67-71 [in Russian].

Siivonen L. 1979. [Mammals of Northern Europe]. Moskva: Lesnaya Promyshlennost'. [In Russian].

Sharova L.P. 1975a. [On variability of common shrew on Southern Ural Mts] // Informatsionnye Materialy IERiG. Sverdlovsk: Institute of Animal and Plant Ecology, Ural
Branch of the Russian Academy of Sciences. P.45-46 [in Russian].

Sharova L.P. 1975b. [Species structure and population diversity of shrews (genus Sorex) in steppe pinery of TransUral and Kazakhstan] // Populyatsionnaya Izmenchivost' Zhivotnykh. Sverdlovsk: Institute of Animal and Plant Ecology, Ural Branch of the Russian Academy of Sciences. P.91-97 [in Russian].

Searle J.B. \& Thorpe R.S. 1987. Morphometric variation of the common shrew (Sorex araneus) in Britain, in relation to karyotype and geography // Journal of Zoology. Vol.212. P.373-377.

Sheffe G. 1980. [Dispersion Analysis]. Moskva: Mir. [In Russian].

Shchipanov N.A., Bobretsov A.V., Bulatova N.Sh., Kalinin A.A. \& Kupriyanova I.F. 2005. Ural chromosomal Serov race of common shrew Sorex araneus L. (Insectivora, Mammalia) that inhabits polydominant deep coniferous taiga in the north of European Russia // Doklady Biological Sciences. Vol.404. P.360-363.

Sulkava S., Vahtola M. \& Fredga K. 1985. Structure of the upper tooth-row of Sorex araneus in Scandinavia // Acta Zoologica Fennica. Vol.173. P.237-239.

Vasil'ev A.G. \& Sharova L.P. 1992. [The geographical and chronographical correlation of common shrew variability on Ural Mtns] // Morfologicheskaya i Khromosomnaya Izmenchivost' Melkikh Mlekopitayushchikh. Ekaterinburg: Nauka, Ural'skoe Otdelenie. P.94-108 [in Russian].

Wójcik J.M., Bogdanowich W., Pucek Z., Wójcik A. M. \& Zalewska N. 2000. Morphometric variation of the common shrew Sorex araneus in Poland, in relation to karyotype // Acta Theriologica. Vol.45. Suppl.1. P.161-172.

Yudin B.S. 1989. [Insectivorous Mammals of Siberia]. Novosibirsk: Nauka, Sibirskoe Otdelenie. [In Russian]. 